\title{
Expectativas de estudantes brasileiros relacionadas a integração, motivação no ensino e aprendizagem autorregulada
}

\author{
Brazilian students expectations related to integration, motivation and \\ self-regulated learning
}

Mara Lazzaretti Bittencourt*, Evely Boruchovitch**

* Comissão Permanente para os Vestibulares, ** Faculdade de Educação. Universidade Estadual de Campinas

\begin{abstract}
Resumo
Neste trabalho, apresentamos os resultados de um estudo sobre expectativas de estudantes realizado em $2015 \mathrm{em}$ uma universidade pública brasileira. Os dados foram coletados por meio do Questionário de Expectativas de Estudantes Universitários (QEXP-U), um instrumento construído na referida universidade após análise qualitativa sistemática do conteúdo de expectativas de ingressantes em anos anteriores. Participaram do presente estudo 1239 estudantes ingressantes e suas respostas foram analisadas por meio das técnicas estatísticas da Análise Fatorial. Emergiram 6 fatores, com bons parâmetros psicométricos, que mostram que os ingressantes têm expectativas positivas e se encontram motivados para o curso universitário. As expectativas revelam que existem, na universidade, condições para uma atuação educativa que promova a aprendizagem autorregulada.

Palavras-chave: questionário de expectativas, ensino superior, integração, motivação, aprendizagem autorregulada.
\end{abstract}

\begin{abstract}
In this paper we present the results of a study about the expectations of university students conducted in 2015 in a Brazilian public university. Data were collected through the University Student Expectations Questionnaire (QEXP-U), which is an instrument constructed in the university after a systematic qualitative analysis of the content of students expectations in previous years. A total of 1239 freshmen students participated in this study. Student responses were analyzed using the statistical techniques of Factor Analysis. Six factors emerged, with good psychometric parameters, which show that the students have positive expectations and that they are motivated for the university course. The expectations show that there are conditions in the university for an educational action that promotes self-regulated learning. Keywords: expectations questionnaire, higher education, integration, motivation, self-regulated learning.
\end{abstract}

Estudos que permitem conhecer características mais específicas dos estudantes universitários, tais como o que eles esperam encontrar e realizar durante os anos do ensino de graduação, assim como suas atitudes, valores, interesses, realizações, crenças e formas de engajamento nas atividades curriculares e extracurriculares têm recebido destaque na literatura nacional e internacional pelo alcance que suas informações trazem para a compreensão dos aspectos que afetam o desenvolvimento do estudante universitário, bem como pelo poder preditivo do ajustamento, da integração e adaptação no contexto acadêmico (Almeida e Soares, 2003; Hu, Katherine e Kuh, 2011; Jackson et al, 2000; Kuh, 2000; Luo e Jamieson-Drake, 2005; MarinhoAraújo et al, 2015; Soares e Almeida, 2004). Pesquisas sobre as expectativas de estudantes universitários, em particular, fazem referência aos diferentes motivos que podem interferir na integração dos estudantes no ensino superior e levar a comportamentos e trajetórias distintas entre eles. Enquanto alguns estudantes se desenvolvem não apenas em termos acadêmicos, mas também nas esferas da vida emocional, psicossocial, social e/ou cultural, outros se mostram despreparados para as mudanças relativas a essa etapa de suas vidas. Vários estudos têm demonstrado que as expectativas podem estar relacionadas a descontentamentos, isolamento social, sintomas de ansiedade, stress, repetência nas disciplinas e a evasão dos cursos (Baker et al, 1985; Brinkworth et al, 2009; Casanova e Almeida, 2016; González et al, 2011; Jackson et al, 2000; Reynolds e Baird, 2010; Shankland et al, 2010).

No cenário educacional atual, os estudos sobre expectativas de estudantes universitários mostram-se particularmente relevantes devido às políticas de acesso ao Ensino Superior que têm se pautado pela promoção da diversidade dos estudantes. Muitas instituições de Ensino Superior estão sendo desafiadas a atender um perfil de aluno mais heterogêneo, seja no que se refere à origem social dos estudantes, à trajetória escolar anterior, à situação de trabalho, às questões raciais. Neste contexto torna-se fundamental que essas instituições conheçam as necessidades e expectativas de seus estudantes para que possam atuar no sentido de propiciar uma integração adequada, inclusive garantindo um ensino de qualidade a alunos com perfil socioeconômico menos favorecido, que constituem o público alvo das políticas de inclusão 
social, não apenas no Brasil, mas em diferentes países que estão vivenciando um movimento mundial de democratização do Ensino Superior (Almeida e Soares, 2003; Soares et al, 2016; Casanova e Almeida, 2016; Hu, 2011; James, 2002; Marinho-Araújo, 2015; Mercuri e Polydoro, 2003; Santos et al, 2013).

Autores conceituados da literatura internacional (Astin,1999; Tinto, 1975) desenvolveram teorias que trazem informações relevantes ao debate sobre as expectativas de estudantes universitários, teorias essas que permitem identificar quais são as diferentes atividades vivenciadas pelo estudante durante o ensino de graduação que concorrem para que a sua integração e retenção no Ensino Superior seja adequada. De acordo com Tinto (1975) e com Astin (1999), a integração ocorre quando há envolvimento dos estudantes em atividades curriculares e extracurriculares, bem como quando existe identificação com a universidade e interação com os docentes, dentre outros fatores. Várias análises, realizadas a partir dos conceitos das teorias desses autores, buscaram identificar a relação entre a participação dos estudantes em atividades extracurriculares e o desenvolvimento do papel de liderança (Foreman e Retallick, 2013); a relação entre o engajamento nas atividades acadêmicas e sociais com a obtenção de notas e os níveis de satisfação com a experiência universitária (Webber et al, 2013); a importância da interação entre estudantes e o corpo docente (Hoffman, 1996). Soares (2016) também apresenta vários estudos que articulam o bom relacionamento entre professor e aluno, a motivação dos docentes para as atividades de ensino e a infraestrutura das instituições com a integração adequada dos estudantes no Ensino Superior. Constata-se, pois, que o processo de integração do estudante e sua retenção no ensino superior têm condições de ocorrer satisfatoriamente, sempre que as expectativas estudantis são positivas e encontrem condições institucionais para sua consecução. Mais recentemente, outros pesquisadores também relacionaram as expectativas mais positivas e realistas dos estudantes, assim como a sua disposição e motivação para o Ensino Superior ao rendimento acadêmico mais satisfatório e à vivência universitária bem sucedida (Soares, 2016; Casanova e Almeida, 2016). Neste estudo, apresentamos as expectativas de estudantes universitários que foram levantadas a partir de um questionário elaborado pelas autoras do estudo e as analisamos à luz da perspectiva da aprendizagem autorregulada.

\section{Método}

\section{Participantes}

Um número expressivo de ingressantes no ano de 2015 participou do presente estudo $(n=1239,37,5 \%)$. Os participantes matricularam-se em cursos das diferentes áreas de conhecimento: Artes, Biológicas, Engenharias, Exatas, Humanas e Tecnológicas, com percentuais de representatividade que variam entre $28 \%$ (área Tecnologias) a 40,8\% (área de Artes), conforme pode ser visto na tabela 1 . Todos os cursos oferecidos na universidade $(n=66)$ estão representados na amostra. No que diz respeito às características gerais, 57,2\% dos participantes são homens $(n=709)$ e $42,8 \%$ mulheres $(\mathrm{n}=530)$. Esses estudantes, em sua maioria, são jovens que têm até 20 anos de idade $(75 \%, \mathrm{n}=991)$, solteiros $(97,2 \%, \mathrm{n}=1204)$ e não exercem atividade profissional (78\%, n=971). Há maior número de estudantes que cursaram somente escolas particulares $(62,15 \%, n=770)$ no ensino médio. Os que frequentaram somente escolas públicas nesse nível de ensino representam 32,2\% $(\mathrm{n}=400)$. É importante destacar que os participantes da pesquisa apresentam características socioeconômicas semelhantes as do total de ingressantes em 2015.

Tabela 1.

Distribuição dos ingressantes e participantes 2015 por área de conhecimento

\begin{tabular}{lccl}
\hline Área & Ingressantes & Participantes & $\%$ \\
\hline Artes & 174 & 71 & 40,8 \\
Biológicas & 585 & 199 & 34,0 \\
Engenharias & 995 & 399 & 40,1 \\
Exatas & 622 & 258 & 41,5 \\
Humanas & 798 & 275 & 34,5 \\
Tecnologias & 132 & 37 & 28,0 \\
Total & $\mathbf{3 . 3 0 6}$ & $\mathbf{1 . 2 3 9}$ & $\mathbf{3 7 , 5}$ \\
\hline
\end{tabular}

\section{Instrumento}

Os dados foram coletados por meio do Questionário de Expectativas de Estudantes Universitários (QEXP-U), um instrumento que se destina aos alunos que ingressam anualmente nos cursos de graduação da referida universidade. Este instrumento é composto por 43 itens em formato de escala Likert, com 6 opções de resposta discordo muito; discordo; discordo pouco; concordo pouco; concordo e concordo muito -, e foi construído após análise qualitativa sistemática do conteúdo de um universo de expectativas de estudantes ingressantes na universidade em 1997, 2005 e 2010. O mapeamento exaustivo e rigoroso das perspectivas qualitativas dos estudantes ingressantes (cerca de 80\% deles) em anos anteriores e o banco de dados gerado a partir desse mapeamento representam aspectos que contribuíram positivamente para a elaboração do QEXP-U. Os 43 itens foram distribuídos aleatoriamente no questionário e dizem respeito às seguintes dimensões de expectativas: Formação para o emprego e carreira; Qualidade da formação; Qualidade das aulas; Envolvimento na vida acadêmica; Desenvolvimento psicossocial; Formação para a cidadania e para o desenvolvimento social e ético; Infraestrutura, organização e apoio aos alunos; Vida social universitária.

\section{Procedimento}

Todos os estudantes que ingressaram na universidade em 2015 foram convidados a participar do estudo. Eles receberam uma mensagem eletrônica, assinada pelo coordenador de pesquisa da Comissão dos Vestibulares, em que eram dadas as boas vindas à universidade, explicitados os objetivos e a relevância da participação. 
na pesquisa. Os alunos foram livres para participar ou não do estudo. Foram assegurados o sigilo das informações pessoais e a confidencialidade das respostas. Os que concordaram em participar da pesquisa, um número expressivo de estudantes (1239, $37,5 \%$ do total de ingressantes em 2015), acessaram um link anexado à mensagem no qual se encontrava o QEXP-U.

O exame das propriedades psicométricas do questionário e de sua estrutura fatorial foi realizado por meio dos procedimentos estatísticos do Statistical Analysis System (SAS), versão 9.2. Foram obtidos bons resultados no que tange à consistência interna do questionário total e de cada fator, estimadas pelo coeficiente Alfa de Cronbach. O Teste de Esfericidade de Bartlett-Kaiser mostrou que há um nível de probabilidade muito adequado para a correlação entre os itens e a amostra, averiguada pelo índice de Kaiser foi considerada apropriada para a realização da Análise Fatorial. Assim, para a identificação da estrutura interna do questionário foram empregados o método dos componentes principais (eigenvalue superior a 1,0) e a rotação Varimax.

\section{Resultados}

Emergiram 6 fatores, com bons parâmetros psicométricos, que revelaram as expectativas dos ingressantes: 1) Desenvolvimento Humano e Profissional e Recursos Institucionais para o Ensino $(\alpha=0,90)$; 2) Formação para a Carreira/emprego e Sucesso Profissional $(\alpha=0,80)$; 3) Interação Social e Sentimento de Pertencimento $(\alpha=0,87)$; 4) Formação orientada à Cidadania $(\alpha=0,78)$; 5) Experiências Formativas que Transcendam a Sala de Aula $(\alpha=0,58)$ e 6) Reconhecimento Acadêmico e Familiar $(\alpha=0,63)$.

\section{Discussão}

A análise dos fatores e dos itens que os compõem mostra que os estudantes ingressantes têm expectativas ricas e diversificadas. Os resultados mostram também que os estudantes apresentam expectativas positivas e otimistas em relação à universidade, ao curso em que se matricularam, às atividades curriculares e extracurriculares, aos docentes e à vida social universitária, o que revela que se encontram motivados para essa nova etapa de suas vidas. Os dados obtidos no estudo trazem ainda outras contribuições relevantes. Eles mostram aos docentes e gestores da instituição que existem condições para uma atuação educativa que promova a aprendizagem autorregulada, visto que os principais atores desses processos, os alunos, explicitam em suas expectativas que desejam receber uma formação que está fundamentada em vários elementos que concorrem para a efetivação desse modo de aprender, seja no que diz respeito ao tipo de formação desejada uma formação para o desenvolvimento pleno do estudante-; no que se refere às expectativas por um ensino fundamentado na aquisição de autonomia para os estudos e no posicionamento reflexivo, crítico e proativo do estudante frente ao processo de aprendizagem; seja porque os ingressantes parecem possuir condições afetivas e motivacionais que concorrem para que a autorregulação da aprendizagem se efetive (Boruchovitch,2014). Como a literatura da área relaciona as expectativas mais positivas e otimistas dos estudantes, assim como a disposição e motivação destes para a aprendizagem, com melhor rendimento acadêmico e à vivência universitária bem-sucedida, pode-se dizer que esses ingressantes estão mais propensos a uma integração adequada no Ensino Superior, sobretudo se bem estimulados.

\section{Referências}

Almeida, L. S.; et al. Acesso e sucesso no ensino superior em Portugal: questões de género, origem sócio-cultural e percurso académico dos alunos. Psicologia: Reflexão e Crítica. Porto Alegre, RS, v.19, n.3, p.507-514, 2006.

Disponível em:<http://www.scielo.br/pdf/prc/v19n3/a20v19n3.pd f>. Acesso em: ago. 2016.

Almeida, L. S; Soares, A. P.. Os estudantes universitários: sucesso escolar e desenvolvimento psicosocial. In: MERCURI, E.; POLYDORO, S. A. J. Estudante universitário: características e experiências de formação. Taubaté: Cabral Editora e Livraria Universitária, 2003, p. 15-40.

Astin, A. W.. Student Involvement: A Developmental Theory for Higher Education. Journal of College Student Development, Baltimore, v.40 n.5 p.518-529 Sep-Oct 1999 (1984). Disponível em:< http://kvccdocs.com/KVCC/2013-Spring/FY125-OL A/content/L-17/Student\%20Involvement\%20Article.p df>. Acesso em: 28 nov. 2014.

Boruchovitch, E.; Autorregulação da Aprendizagem: contribuições da psicologia educacional para a formação de professores. Revista Quadrimestral da Associação Brasileira de Psicologia Escolar e Educacional, set-dez 2014, Vol.18 n.3, pp. 401-40.

Brinkworth, R.; et al. First Year Expectations and Experiences: Student and Teacher Perspectives. Higher Education, Ohio, v. 58, n. 2, p. 157-173, 2009. Disponível em:< http://www.jstor.org/stable/40269175>. Acesso em: 14 out. 2015.

Casanova, J.; Almeida, L.S.. Diversidade de público no ensino superior: antecipando riscos na qualidade da adaptação e do sucesso acadêmico em estudantes do $1^{\circ}$ ano. Psicologia, Educação e Cultura, v. 20, n.1, p. 27-45.2016. Disponível em:< http://pec.ispgaya.pt/edicoes/2016/PEC2016N1/index. html>. Acesso em: ago. 2016.

Foreman, E. A.; Retallick, M. S.. Using Involvement to Examine the Relationship between Undergraduate Participation in Extracurricular Activities and Leadership Development. Journal of Leadership Education, v.12, n.2, p.56-73, 2013. Disponível em:<http://www.journalofleadershiped.org/index.php/ vol-13-iss-2>. Acesso em: 05 dez. 2014.

González, M. A.; Gazo, P. F.; Fonseca, M. T.. La Problemática de la Transición Bachillerato Universidad en la Universidad de Barcelona. REOP, Madrid, v. 22, n.1, p 15-27, 2011. Disponível em:< 
http://revistas.uned.es/index.php/reop/article/view/74/ 63>. Acesso em: 18 abr. 2016.

Hoffman, J. A.. Applying Astin's Involvement Theory to Student-Faculty Interaction. Journal of Student Affairs, Colorado, v.5, n.1, p. 10-16, 1996. Disponível em

$:<$ https://dspace.library.colostate.edu/handle/10217/180 77? show=full $>$. Acesso em: 14 nov. 2014.

$\mathrm{Hu}$, Shouping; Katherine, Lindsey; Kuh, George D.. Student typologies in higher education. New directions for institucional research, v. 2011, p. 5-15, dez. 2011.

Jackson, L.M.; et al. Great expectations: the relation between expectancies and adjustment during the transition to university. Journal of Applied Social Psychology, v.30, n. 10, p.2100-2125, 2000. Acesso em: 17 fev. 2014.

James, R.. Student's changing expectations of higher education and the consequences of mismatches with the reality. OECD: Responding to students expectations. Paris: OECD Publications Service, 2002, p.71-84.

Kuh, G.. They shall be known by what they do: an activities-bases typology of college students. The Journal of College Student Development, v.41, n.2, 2000 .

Disponível em:<http://www.articles.com/p/articles/mi_qa3752/is _200003/ai_n8882137/print?tag=artBod $>$. Acesso em: 6 out. 2008 .

Luo, J.; Jamieson-Drake, D. Linking student precollege characteristics to college development outcomes: the search for a meaningful way to inform institutional practice and policy. Association for Institutional Research. Enhancing Knowledge. Expanding Networks. Professional Development, Informational Resources \& Networking, v.7, p.1-16, november, 2005. Disponível em htpp:// www.airweb.org/page.asp?page $=266$. Acesso em: 1 out.2008.

Marinho-Araujo, C.M; Fleith, D. De S.; Almeida, L.S.; Bisinoto, C.; Rabelo, M.L.. Adaptação da escala expectativas acadêmicas de estudantes ingressantes na educação superior. Avaliação Psicológica, v.14, n.1, pp. 133-14, 2015. Disponível em:< http://pepsic.bvsalud.org/scielo.php?script=sci_arttext \&pid=S1677-04712015000100016>. Acesso em: 01 jun. 2016.

Mercuri, E.; Polydoro, S.A.J.. Evasão no ensino superior: algumas contribuições. Estudante universitário: características e experiências de formação. Taubaté: Cabral Editora e Livraria Universitária, 2003, p. 219-236.

Reynolds, J R; Baird, C. L.. Is There a Downside to Shooting for the Stars? Unrealized Educational Expectations and Symptoms of Depression. American Sociological Review, v. 75, n. 1, p. 151-172. 2010. Disponível em:<http://www.jstor.org/stable/27801515>. Acesso em: 11 set. 2015.

Santos, A. A. A. Dos.; Polydoro, S.A.J.; Scortegagna, S.A.; Linden, M. S.S.. Integração ao ensino superior e satisfação acadêmica em universitários. Psicologia:
Ciência e Profissão, v.33, n.4, p. 780-793, 2013. Disponível em:< http://www.scielo.br/pdf/pcp/v33n4/v33n4a02.pdf > Acesso em: 29. Mar. 2016.

Shankland, R. et al. Student Adjustment to Higher Education: The Role of Alternative Educational Pathways in Coping with the Demands of Student Life. Higher Education, Ohio, v. 59, n. 3, pp. 353-366, 2010. Disponível em: <http://www.jstor.org/stable/25622188>. Acesso em: 10 set. 2015.

Soares, A.B; Monteiro, M.C.; Porto, A.M.Da S.; Gomes, G.; Gomes, C.A.O.. Expectativas acadêmicas de estudantes de psicologia brasileiros: estudo comparativo entre iniciantes e concluintes. Revista de Psicologia, Educação e Cultura, v.20, n.1, p.46-64, $2016 . \quad$ Disponível em:< http://pec.ispgaya.pt/edicoes/2016/PEC2016N1/index. html>. Acesso em: ago. 2016.

Tinto, V.. Dropout from Higher Education: A Theoretical Synthesis of Recent Research. Review of Educational Research, v.45, n.1, p.89-125, 1975. Disponível

em: <http://www.jstor.org/stable/1170024?seq=1\#pag e_scan_tab_contents>. Acesso em: 15 out. 2015.

Webber, K. L.; Krylow, R.B., Zhang, Q.. Does Involvement Really Matter? Indicators of College Student Success and Satisfaction. Journal of College Student Development, v.54, nº, p.591-611, 2013. Disponível em: <https://muse.jhu.edu/article/528367>. Acesso em: 01 jul. 2015. 